\title{
VIRTUAL REALITY AND CAPTURED REALITY FOR CULTURAL LANDSCAPE COMMUNICATION
}

\author{
Daniele Calisi ${ }^{1}$, Stefano Botta ${ }^{1}$ \\ ${ }^{1}$ Dipartimento di Architettura Roma Tre, Italia, daniele.calisi@uniroma3.it, stefano.botta@uniroma3.it
}

Commission II

KEY WORDS: Virtual Reality, Captured Reality, Survey, Cultural heritage, Communication

\begin{abstract}
:
In the last twenty years, the process of digitization of art and cultural heritage in general has undergone considerable developments pursuing purposes of conservation and analysis, first, but also of dissemination and knowledge of the works themselves or of the sites of historical - archaeological - architectural interest. For years, museums and archaeological sites have begun to digitize their assets to make them usable on online platforms. In this way, multiple high-resolution digital archives and virtual reconstructions of the sites were born that provide immersive experiences to the user. Above all, the latter stimulate the cinematographic and videogame industries to continuous research to improve the immersive and interactive experience, thanks to the use of virtual reality devices applied to increasingly detailed digital models, in some cases obtained thanks to the most modern detection instruments.
\end{abstract}

\section{INTRODUCTION}

For many years the instrumental survey, from photos in SfM or with laser scanners, was almost exclusively limited to the archaeological and architectural sphere, that is to say on the historical and cultural heritage that characterizes a specific place. However, the latest research, also by virtue of the guidelines indicated by the SNSI and PNR, tend to place increasing interest in the cultural landscape, understood as a semantically complex set of material and immaterial entities deriving from anthropic interaction on the natural context. This system is made up of relationships between man and the environment that surrounds him and consequently of how the individual transforms it based on personal perceptions and actions.

Clearly it is a completely subjective system of a specific society that lives in a specific place, its habitat, establishing ever different relationships, according to a singular and indigenous language, highlighting the complexity in studying the infinite systems of each single circumscribed area.

It follows that the survey and the representation of this union of the cultural landscape are extremely complicated, not only from a technical point of view of shooting, but also and above all of fruition of the data obtained. Communicating the cultural landscape means trying to translate that particular language made up, as mentioned, of relations between several elements in communication with each other.

To cope with such a complex system, it has become necessary to use increasingly advanced detection techniques, often interlaced with each other, which allow the acquisition of as much data as possible in view of subsequent processing, discretization and communication (Battini, 2017).

The combination of terrestrial and aerial survey techniques such as photogrammetry, laser scanner, Lidar, and SfM, is the essential support for an increasingly detailed and compliant acquisition of complex sites of interest, consisting of physical and naturalistic components. The $3 \mathrm{D}$ reconstructions thus produced are strictly faithful to reality, so much so that the technique is defined as Captured Reality.

The models obtained and the related elaborations have been used for years in many sectors, initially analytical and conservative, but more recently as virtual scenarios in videogames, serious games or films, carefully elaborated and mixed with special effects in order to recreate realistic scenarios in where an immersive experience is possible through the latest communication techniques of Virtual Reality and Augmented Reality (Greengard, 2019).

In the last two years, travel restrictions due to the pandemic emergency have accelerated many processes of digitization, communication and use of an ever-increasing number of sites of historical and artistic interest, as well as archives and libraries, dynamically expanding the possible combinations of acquisition, management and return of the data to be scanned.

The examples that we report, in summary form, allow us to better understand the direction and guidelines, at a global level, that are being adopted to pass on, with innovative techniques, the tangible and intangible cultural heritage.

\section{RELATED WORKS}

Some of the examples, analysed for research purposes, reveal several fundamental aspects on the interaction between instrumental survey and the last frontiers of communication through VR.

A first case study concerns a research conducted by the Center for Research Creation (CRCDM) at Sunway University in Kuala Lumpur in Malaysia, which focused on the disappearance of indigenous tangible and intangible heritage, as well as on a new approach to raise awareness through immersive experiences by the user, through the use of digital media. Visitors can learn about a variety of engaging content that includes unique examples of Malaysian cultural heritage, fiction and craft traditions. The research moves the steps from a fundamental awareness: if the tangible heritage can, from a certain point of view, easily acquire, the intangible heritage, on the other hand, is not so quick or easy to document.

The latter is the "soul" that accompanies the material heritage, it can be described as "aura" (Latour et al., 2011): an object can be created as part of a ceremonial apparatus, or a place where a cultural activity takes place, or as a tool to document oral traditions in a culture. Therefore, beyond its formal aspect, the meaning that an object has assumed in history is just as important and requires the right tools for the right disclosure, sensitizing the new generations to the folk culture which is part of a past heritage, but which does not belong forgotten. 
With this in mind, CRCDM has worked on several fronts with various digital technologies. It recreated a Multiscreen Malaysian Folklore story-telling exhibit. It collected the stories handed down orally by the Mah Meri culture (mask carving) and transposed them into comics with Augmented Reality application to better explain a practice that is fading.

In it each traditional mask represents a spirit with an ancient history transmitted from one generation of Mah Meri sculptors to the next.

The same AR was used to explain the designs contained in the traditional sacred weaves called Pua Kumbu, hand made by Iban women in the Sarawak jungle.

Similarly, the intangible heritage of the construction techniques used by Mr. Goh, who is the only Hainan boat builder on the island of Pangkor, has been documented. The research team produced a documentary video, a 12-minute documentary version of the exhibit and an augmented reality book about how the 70-year-old master boat builder builds his fishing boats. All digital data was curated in forms that could be easily exhibited to the public.

Finally, as an additional final product, they advertised a conceptual digital platform for immersive virtual reality called The Hidden Waterfall City. A kind of container that would allow the viewer to be interested in the serious game technique, and within which the partially described products have converged. This project also presented the development of a virtual reality (VR) cultural heritage experience on a local scale, embodied in a transmedia storytelling approach to showcase VR museology content in a public setting.

The Makam Sultan Hussin Shah is an ancient sultan's tomb presented in a VR Museum Experience in the form of a threedimensional model reproduced using photogrammetry to scan the current heritage site of Malacca and set in a conceptual reconstruction of the landscape in which it had to be insert.

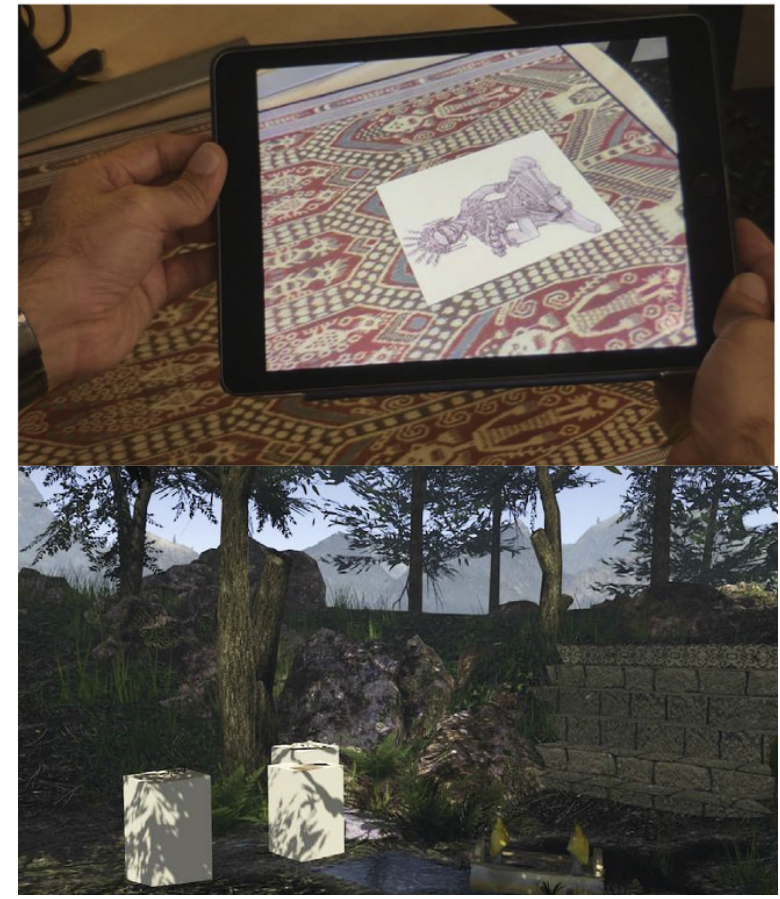

Figure 1: A research conducted by the Center for Research Creation on the tangible and intangible heritage to be communicated through AR and VR. Above sacred weaves called Pua Kumbu viewed in Augmented Reality. Below the Makam Sultan Hussin Shah, an ancient sultan's tomb presented in a Virtual Reality.
A second emblematic case study is represented by the study conducted on the archaeological site of Sarmizegetusa Regia (Romania), where an "open world" visit was created by combining photogrammetry with a video game graphics engine (Demetrescu et al., 2019). The aim of the CNR-ITABC research group was to investigate the potential of Virtual Reality in communicating the characteristics, even intangible ones, of the cultural landscape in the form of digital replication.

The Colonia Ulpia Traiana Augusta Dacia Sarmizegetusa (108$110 \mathrm{AD})$ consists of unique and interesting archaeological remains of an imperial city with a castrum structure with buildings of particular importance such as the Forum, an Amphitheater, the Domus Procuratoris and temples for worship. Destroyed by the Goths, the city lost its original characteristics, and the area took on new meanings, mostly landscape, with crops, pastures and wooded areas.

Most of the ancient city is still underground, and the remains unearthed so far alternate with the semantic entities typical of agricultural land, from paths to fences, from farms to indigenous rural buildings, making it clear how, today, the archaeological heritage is inseparable from the social-landscape and ethnoanthropological aspects of the place. All the aspects of this complex system had to be detected and acquired: the ruins, the spontaneous and cultivated vegetation, the rural accessories, but also the natural elements such as the quality of the light or the herbaceous and arboreal types. To achieve the purpose of such a complete acquisition of digitized data, the research group worked with different techniques on different fronts: a photomodelling derived from an accurate photographic campaign from the ground and with large-scale UAV drone of several parts of the site, united in post-production thanks to the use of targets and GPS systems with unique coordinates; an accurate survey of local light, through the creation of HDRIs with the mirrored ball technique photographed with different values of display spots, then used for the lighting of the virtual model; survey of the vegetation through the sampling of herbs, leaves, fruits or barks, for the creation of a library useful for the modelling of naturalistic essences with appropriate randomization of the elements to avoid the repetition of the scanned entities; finally, virtual reconstruction for the elements that are difficult to make using the SfM technique. This approach has allowed the development of a library of natural and anthropogenic resources to be placed in the setting, further enhancing the simulation in VR.

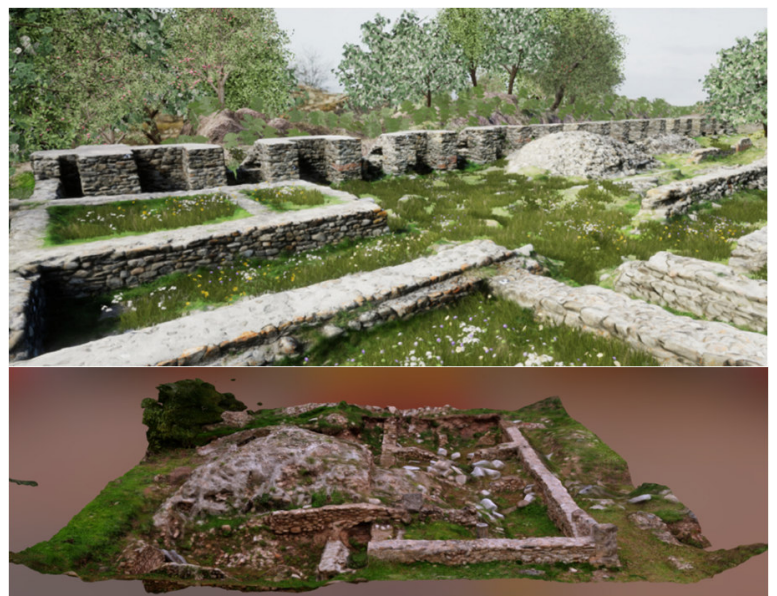

Figure 2: The archaeological site of Sarmizegetusa Regia (Romania). The site was subjected to a scan of the tangible and intangible heritage, from archaeological remains to rural elements, from naturalistic elements to those relating to lighting, to ensure an immersive digital experience as much realistic as possible. 
The Mixed Reality experience was produced through the use of Unreal Engine 4 software, creating an immersive reality of the digitized archaeological and landscape site to be explored with VR viewers. The elements were inserted, using the same reference system, from time to time within the platform, digitally recomposing the Sarmizegetusa site and its rural and naturalistic context, all illuminated by HDRI. The immersive experience allows the user not only to navigate the area by discovering the remains and objects inserted from the excavations, but also to access multimedia information in multiple languages describing the historical-archaeological and ethnological aspects. Sarmizegetusa's case study is of undoubted interest, because it attempts to transmit as much information as possible into the digital environment. However, the aspects of the landscape linked to the other senses still remain to be developed, introducing synaesthetic phenomena that connect different sensory experiences: the temperature of an environment through colour, the tactility of a surface through sight, or the motion of the wind thanks to its sound (Riccò, 2008).

These two examples analysed, together with the research conducted by IUAV and Ca 'Foscari, of the submerged wreck of the Mercury (Belletti et al., 2016) or the virtual museum of the Regolini-Galassi Tomb in Cerveteri (Petroni et al., 2014), highlighted the importance of intangible heritage, which was often not considered, especially in surveys aimed at mapping materials and decay and restoration. Today, the opportunities for acquiring cultural heritage are diversified and also incorporate possible immersive experiences in VR, which cannot ignore the context and the intangible aspects of the heritage, pushing research to find new ways for communicating the acquired data.

\section{THE TEMPLE OF VALADIER IN GENGA}

A case of significant interest in the cultural heritage sector is the Temple of Valadier in the Gola della Rossa in the municipality of Genga, where the historical and architectural significance is combined with a unique and unrepeatable landscape heritage In fact, it is an area of extreme geological and naturalistic interest, of karst formation, which incorporates the wonderful scenery of the Frasassi caves. The small temple of Valadier is located at the entrance to the Grotta della Beata Vergine, located at $319 \mathrm{~m}$ above sea level, one of the most important cavities in the Frasassi Gorge system, due to its use in prehistoric and historical eras. The small temple coexists in this environment with the hermitage of Santa Maria Infra Saxa, dated to the year 1029, whose access was more natural, jagged and lowered, then radically modified centuries later, levelling the walkable altitude precisely to allow the construction of the Temple in 1828, commissioned by Pope Leo XII. Despite the name, which assumes a project by Giuseppe Valadier, the temple was actually designed in two phases, first by Pietro Ghinelli, and then later by Tommaso Zappati and Carlo Donati di Spoleto. Valadier was the most experienced supervisor and advisor and probably revised Ghinelli's design, influencing the final project. It is no coincidence that the style of the temple recalls the neoclassical forms of the architecture of Valadier himself, with a travertine cladding, obtained from the quarry on the slopes of the mountain of the Frasassi caves complex, and with a dome lined with lead plates.

The complex described is an indivisible unicum of architecture, symbols and landscape, impossible to detect only in basic geometries. The complexity of the natural shapes of the karst cave, its colours and lighting guided the choice of a survey based on $\mathrm{SfM}$ techniques, through a photographic project of 48 stations, in which multiple shots were taken to form spherical portions with overlapping of the images by $50 \%$, for a total of 789 photos. The greatest difficulty in this context was to be able to take photographs as close as possible to each other, from the point of view of exposure and contrasts between lights and shadows. However, an intervention in post-production of the *.raw images was necessary, lightening the too dark areas and recovering the burnt ones, which would guarantee a greater match of homologous points in the virtual reconstruction in the software. The greatest attention was paid to the external and internal shots of the cave and by adequately calibrating the filter images between the two indispensable environments to ensure the outside/inside connection and the entirety of the reconstructed virtual model.

The resulting dense point cloud is of excellent quality, with 15 million points, and double number of triangles mesh, although limited to the area that can actually be visited. A drone flight would have guaranteed a greater level of contextualization, despite the dense vegetation would have been an obstacle to a good data acquisition. In analogy with the case studies presented, the contextualization was entrusted, as we will see, to a virtual reconstruction of the orography of the place, with the insertion of the model obtained for SfM, and digital modelling of the vegetation.

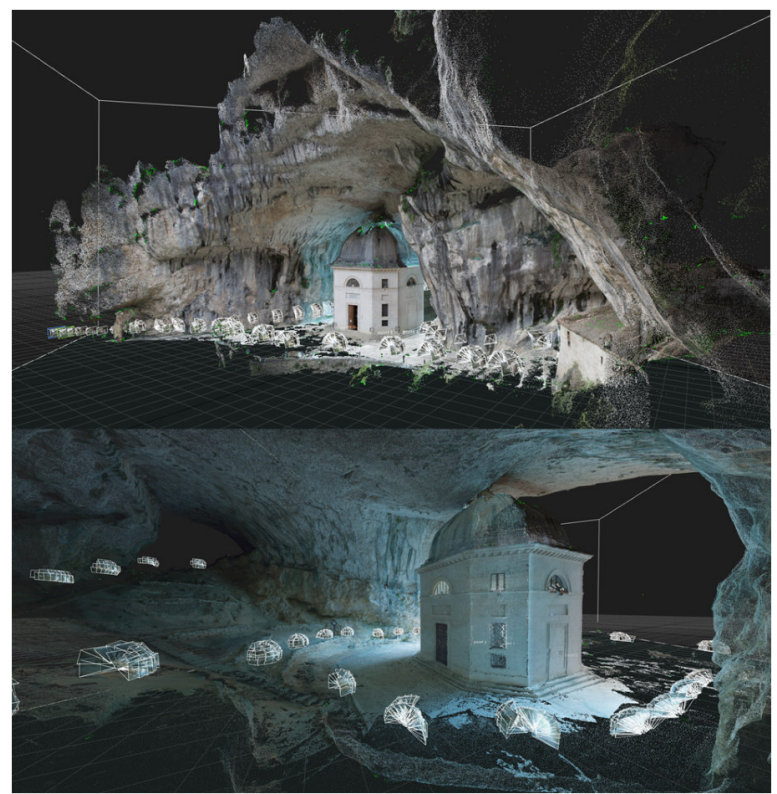

Figure 3: The cave of the Blessed Virgin in the Gola della Rossa, at the Frasassi Gorge system. Virtual reconstruction using the $\mathrm{SfM}$ technique, with 48 positions and 789 shots to form portions of spherical shell.

The planimetric layout of the temple is octagonal, but irregular, with equal sides four by four. According to the latest geometric analyses, this shape is obtained starting from the longer side, the main one and the entrance to the temple. Having built the square on this side, it is possible to obtain a sequence of harmonic rectangles based on the side $\sqrt{2}$ and $\sqrt{3}$, respectively diagonals of the first square and of the first rectangle obtained. But these proportions are also exactly those found in the star of Solomon, or star of David, which is ultimately at the basis of the geometric construction of the plan of Temple of Valadier (Calisi et al., 2019). Similar geometries are also found in the elevation where the temple is contained within an equilateral triangle with a base adjacent to the ground line. The lantern touches the sides of the triangle, while a harmonious rectangle determines the width and height of the façade. Beyond the purely geometric layout, the identified buildings also conceal meanings in analogy with the place where the temple is inserted, highlighting that system of 
intangible entities that needs to be acquired and transmitted through today's technological communication.

The meeting of the two triangles symbolizes the perfect union of spirit and material: the spirit, personified by the temple, by the church, and material, that is the nature which, for those who have been in the Gola della Rossa, reigns supreme and uncontaminated in the 'hermitage.

But on the other hand, everything that exists in nature and manifests itself in the phenomenal world is the reflection of the invisible world of the causes that exist in the beginning. It follows therefore, in that one geometric symbol, that microcosm and macrocosm interpenetrate each other, divided and united at the same time.

The divine geometric rule (the triangle of the holy trinity, the star of David) opposed to the natural force, God and all that he created.

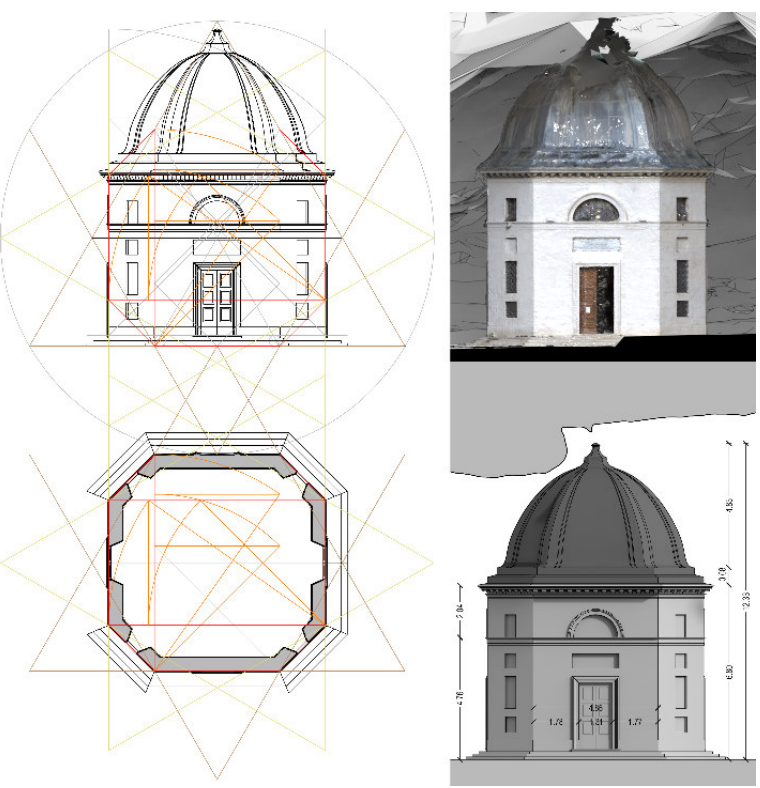

Figure 4: Mesh processing of dense point cloud from SfM. Geometric reconstruction of the temple based on harmonic rectangles and David's star. Virtual NURBS reconstruction.

The information acquired on the study site also concerns the archive drawings (although only of old excavations carried out inside the cave and in any case incorrect if superimposed on the instrumental survey), the historical information of the hermitage, the artistic emergencies (the temple has a copy of a marble statue of the Madonna with child by Antonio Canova), the orographic surveys on the maps and research of DEMs, the acquisition of audio files in several locations, as well as the analysis of the arboreal essences and native flora to be recreated in digital environment.

A considerable amount of information that needed to be communicated through a more complex system than canonical two-dimensional drawings only.

Spatial scans using laser scanners or SfM techniques have had a greater field of application in formal and geometric, material and degradation analyses, mainly through $2 \mathrm{~d}$ redesign and orthophotoplanes of point clouds or textured mesh models (De Luca, 2011). However, in the last two years, the pandemic emergency has further highlighted the possibility of exploiting these techniques to allow digital communication and therefore the remote accessibility of information and places accurately scanned digitally. Research in this sense has made numerous steps forward and the institutions, museums and sites that have introduced virtual tours, in different ways, on their portals have multiplied.

The study site, in addition to the contingent access difficulties, had, in fact, all the characteristics to be disclosed through an immersive digital platform, which allowed users to be able to navigate and understand the many peculiarities, material and intangible, of the Grotta della Beata Vergine. All the information acquired, therefore, was managed and organized on the Unreal Engine 4.27 platform to return the user a product that can be navigated from any desktop or mobile device, ensuring its full navigability.

\section{DEVELOPED METHODOLOGY}

During the reconstruction of the Temple of Valadier virtual scenario, photogrammetric modelling started by using legacy data, made for a previous terrestrial survey. This caused the necessity to make some technical choices to answer themes and difficulties imposed by Virtual Reality. Due to the lack of an aerial survey, made by using drones, it wasn't possible to completely replicate some elements like the floors, difficult to be captured from the ground; after the processing, the Temple's roof shown some missing areas as well, due to a lower visibility or underexposure of several spots, caused by the strong contrasts between the inside and the outside of the cave. Furthermore, a digital survey to rebuild the land of the gorge and the opposite side would have been problematic, even with more advanced tools (Monti et al., 2015). For these reasons, photomodelling and hand modelling were merged where it was necessary, starting from geometrical reconstructions and cartographies to obtain results that are as faithful to reality as possible. In the same way, the vegetational system was later recreated by using libraries of pre-existing models, as it would have been difficult to reproduce it in Captured Reality; the replica was based on a documentary and "in situ" analysis about the main species of plants living in the area.

Aiming at simulating an immersive and engaging experience of the environment surrounding the Temple of Valadier, skylight conditions were later captured to recreate an atmosphere close to the original. The tiled photographs technique was used to do so, taking a series of pictures from a single fixed point of view, targeting several directions (Calisi, 2015). For every position, the operator must take various photos at different levels of exposure, which complicates the following process to reconstruct the final panoramic image. However, there are many softwares born specifically to perform this stitching operation on pictures. The photographic approach was similar to the survey, aiming to guarantee a good level of overlapping between images. A point close to the balustrade was chosen as shooting station, such that it was as far out the cave as possible, in order to obtain a greater portion of the sky. The single photographs were then sewn together thanks to the automated operation "Merge to HDR Pro", available on Photoshop: the sets of pictures with different exposure were uploaded and analysed by the software, which reunited them in a single panoramic HDRI, giving the possibility to make advanced corrections on details, tones and curves. Once modified and reworked, the result was exported to be used as a light source in rendering softwares, with excellent outcomes.

To recreate the soundscape of the Temple of Valadier, a series of audio tracks were registered in several places of the environment, in order to capture and then express the spatialised sound variations between the outside and the inside of the cave. To do so, the choice was to use a ZOOM H4m Pro, which is a stereo digital recorder with adjustable condenser microphones, mounted on a tripod. This device was created for a faithful and clean ambient recording of music and sound effects, guaranteeing versatility and low noise levels. The first track was captured 
inside the cave, the second in one of the two connecting side corridors, the third in the open space in front of the entrance of the Temple. The last track was recorded as close to the balustrade as possible, directing the microphones towards the landscape of the gorge, and using a sound absorbing screen to try to capture only the natural environment outside the site.

The development of the photogrammetric model was carried on the software Reality Capture. The selected and modified images were imported and aligned to generate a first point cloud. The definition of control points on three different edges of the Temple of Valadier was necessary to obtain the collimation among the various parts of the model. The creation of the mesh was then set to a medium detail level, to create a model with a good resolution and preventive excessively heavy data at the same time, simplifying its usage in later stages. The surface produced was made of 23 parts, for a total of 30 million triangles. Right after a manual cleaning process of superfluous areas and elements, the mesh was subdivided in two fundamental components, the Temple and the cave. Those underwent a retopology stage, with different parameters, to simplify and smooth the surfaces and reduce the excessive quantity of data, hardly manageable in realtime softwares with the resources available. Nevertheless, the process tempted to maintain a good level of detail, especially through the textures elaborated afterwards. The final components, reduced and properly textured, counted 5-7 million triangles each.

In the following stage, partially or totally missing elements were hand modelled using the software Rhinoceros (Fig. 5). The roof of the Temple of Valadier was reconstructed starting from the analysis and geometric redrawing of the shape of its faces, and then substituted to the incomplete photogrammetric surface. Similarly, the balustrade, which delimits the path and the view from the cave towards the gorge, was modelled thanks to the support of cartographies, contemporary photos and fragmentary pieces of the mesh, which were eliminated afterwards. As for the floors, the geometries on the ground were first redrawn using an orthophoto coming from the sectioned photogrammetric model; even if partial, it provided enough visual data about the material scanning of the surfaces.

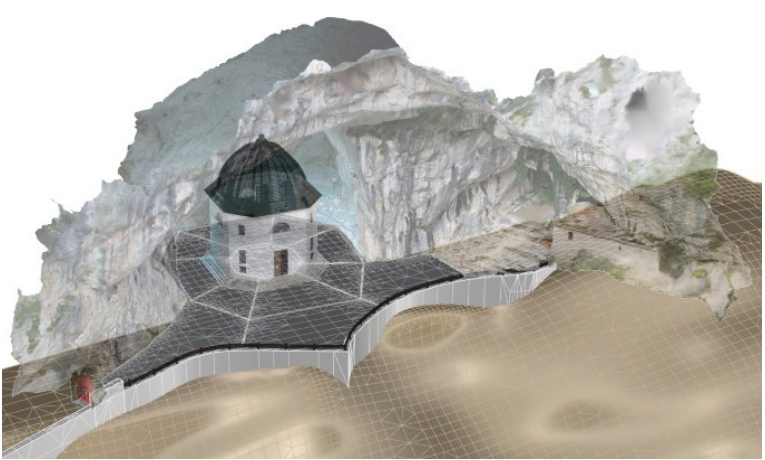

Figure 5: The hand modelled elements, like the floors, the roof of the Temple, part of the terrain and the balustrade, aligned with the photogrammetric surfaces.

Separately, the cave was imported in the software to realize the surface of the floor, starting from the profile obtained from the intersection between the ground and the rocky walls, properly following the variable hight of the perimeter. The surface was then subdivided according to the original geometric drawing to reproduce the material differences. Other additional elements, like the closed gate at the beginning of the walk, were modelled to enrich the scene and functionally support the experience in Virtual Reality.
As for the reproduction of the surrounding terrain, it was not possible to choose the photogrammetric method. In this case, hand modelling was pursued using a cartographic basis, like the one realized by the Parco Naturale Regionale della Gola Rossa e di Frasassi (Fig. 6), compared to the altimetry of Google Maps and other historical photos. This way, it was possible to define a valid hypothesis of the slope below, recreated in depth as it is close to the scene, and terrain on the opposite side, simplified because it was only meant to be a distant background for the virtual setting.

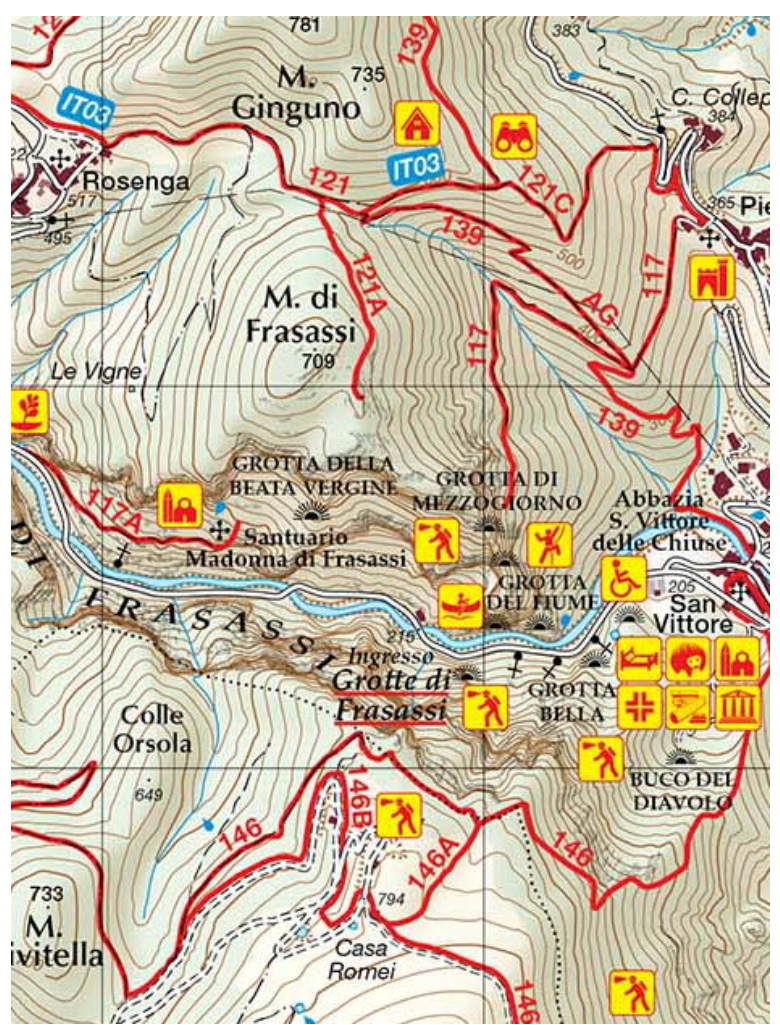

Figure 6: Part of the cartographic reference made by the Parco Naturale Regionale della Gola Rossa e di Frasassi.

The main purpose of the experimentation is to find a new communication paradigm for cultural and landscape heritage, capable of a narrative which holds entertainment and information together, attracting users and pushing them to explore the virtual environment. The perceptual and emotional involvement of the digital experience can be seen as a tool to convey and imprint information the best way. Balancing the two components of the realized experience, the Dionysian one (the ludic, autonomous and chaotic approach) and the Apollonian one (the didactic, directed and linear approach), allows to create an alternative reality with a layered storytelling for the cultural and landscape heritage, giving the user the possibility to choose how to discover its story (Butcher, 2017). This is possible thanks to the use of videogame expedients, such us the inclusion of tasks or fictional stories, hiding elements in the environment to stimulate the research. As a matter of fact, interactivity and immersion are the most important parameters defining a Virtual Reality experience, together with the simulation itself (Bolter, 2000). If realism is already a quality referable to the use of the Capture Reality, the simulation of the scenario can be deepened by using real-time graphic and sound effects to enhance the sense of proximity to physical reality. Being a psycho-perceptual experimentation of a virtual place, 
the presence of a larger number of sensory stimuli helps the user to feel part of the environment, reaching a deeper level of embodiment corresponding to a stronger participation (Riva et al., 2019). The inclusion of active and passive interactions enhances the engagement with the simulation as well, supporting a better understanding of the information transmitted.

To realize the virtual experience of the replicated landscape, the choice was to use Unreal Engine 4.27, a graphic engine created primarily for videogames. With its preinstalled libraries and templates, the software gives an excellent starting point for developing a scenario, requiring only a small amount of programming skills to generate customized events and actions. The transition to the virtual environment was carried out in stages, involving some intermediate steps to speed up and improve the results. The cave and the surrounding scenery were imported separately into the real-time rendering software Twinmotion 2021. The vast library of plants and mineral elements compatible with Unreal Engine (as they are programs from the same developer, Epic Games) makes this transitional stage essential to easily work on the complexity of the natural scene, as well as the presence of tools to manipulate shapes and materials of the terrain.

From the previous analysis on the typical vegetation of the Gola di Frasassi area, a substantial complexity of diversified ecological conditions emerges (Regione Marche, 2000). Quercus ilex (holm oak) and Fagus sylvanica (beech) are two of the most common trees, with the presence of isolated cases of other species such as the Celtis australis (hackberry). The presence of shrubs and flowers, both conventional and rare, is also rich: there are numerous Arbuntus unedo (strawberry tree), Rubia peregrina (wild madder) and Asparagus officinalis (asparagus), as well as multiple varieties of cyclamen and bluebells.

Some of them, such as the trees, were already included in the software archive; as for others, it was decided to select models that were the most similar to the originals, in order to make the surrounding landscape plausible, even if far from the viewer. Once the models were selected, they were scattered on the surface of the ground using a brush tool that allows to randomly arrange multiple species at the same time, rotating, scaling and modifying each element to make them look apparently different, and therefore more realistic (Fig. 7). The operation was carried out separately for trees, flowers and shrubs, in order to better control their location and density. The introduction of grass was postponed to a subsequent application on Unreal Engine, as some problems had already been encountered with the export process during previous experiments. Using the Paint Terrain tool in the Landscape section, the terrain was then textured and detailed to look heterogeneous, choosing different materials, including grass, forest terrain, dirt and gravel.

Thanks to specific plug-ins that facilitate a bridge between the two softwares, the model of the implemented scenery was brought from Twinmotion into a new project on Unreal Engine 4.27. The animations of branches and foliage were also automatically imported, contributing to make the environment more realistic and dynamic.

Aiming to create an experience as immersive as possible, it was decided to start a from a first-person template provided by the software, working directly with a correct visual setup and a properly animated character. This helped to better simulate the perspective of the viewer and would make it possible the subsequently implementation of the stereoscopic vision to optimize the use of the application through HMDs.

The model of the Temple was then imported and placed in its correct position to complete the scene. At this point, the development moved on to the definition of the technical and interactive aspects of the project. First, a collision system was defined for the main surfaces of the scene, designed to simulate the physical restrictions of the objects. The elements forming the user interactive space, which goes from the bottom of the cave to the path in front of the gorge, were characterized by complex collisions, which reproduce the actual limits of the surfaces based on their meshes. The vegetation and the terrain outside, excluded from the active area, were not characterized by these systems to avoid unnecessary slowdowns of the software. In addition, to prevent users from leaving the designated area and falling out of the virtual space, Blocking Volumes were used to better define and manage the space of the active virtual experience.

The vegetation was then implemented with a selection of herbs and flowers, applied to the ground in a scattered way, using the Foliage tools and some open-source models acquired from the software marketplace. In order to optimize the effect, grassy areas were concentrated close to the cave and on the opposite side of the gorge, in the regions where the vegetation was sparser.

To simulate the atmospheric conditions of the place in a realistic way, the HDRI developed from the in-situ photographs was used to illuminate the scene. The image was applied as a map to the HDRI Backdrop element/plugin, which projects the captured real sky into the virtual environment, using it as a primary light source. To accentuate the effect, a direct light was still maintained in the exact position of the real sun, to better simulate and control the shadows. In this case, having manually recreated the background of the Gola di Frasassi, the HDRI was necessary just for a plausible replica of the light and tonal characteristics of the atmosphere. An Atmospheric Fog element was then inserted to reproduce the surrounding atmospheric density and dissimulate the bottom of the gorge. This made it possible to conceal the boundaries of the digital environment in the distance, making the scene more convincing and suggestive at the same time.

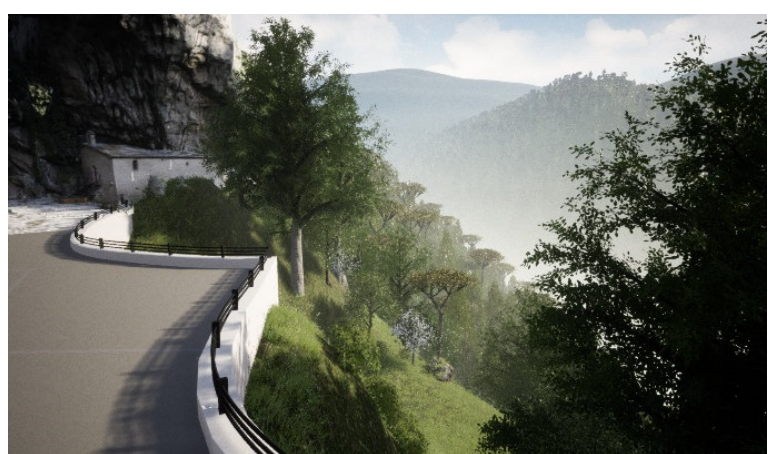

Figure 7: View on the natural landscape of the Gola di Frasassi, inside the simulation in Unreal Engine 4.27.

As for the reproduction of the soundscape, a series of Ambient Sounds were placed where the audio tracks had been recorded. The respective sound was associated with each of the points; then, all the volumes and attenuation rays were set to obtain smooth transitions between neighboring sound environments, improved by the possibility of simulating reverb and attenuation based on the air absorption and the surrounding collisions (Fig. 8). Using proper stereo headphones, it is possible to control the binaural functions, recreating the experience of a sound environment close to the real one, employing sounds to direct the user's attention to specific points. 


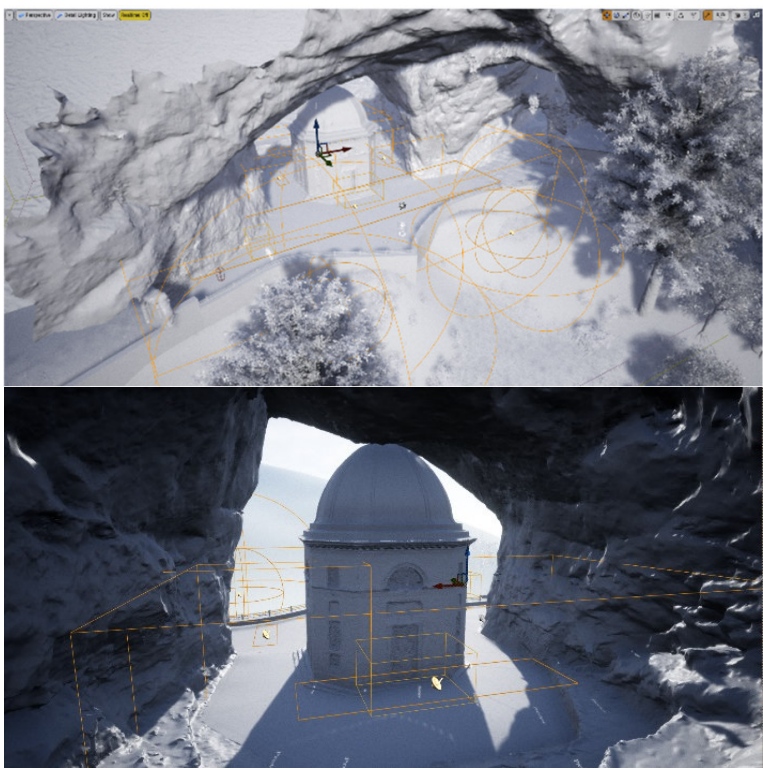

Figure 8: The virtual environment created on Unreal Engine 4.27, in which the Ambient Sounds volumes and the Trigger Volumes have been highlighted.

To integrate a more explicit information component, designed to explain and narrate the place while it is freely explored, a series of description boxes and images were arranged in the salient locations of the site (Fig. 9); they were programmed, using Trigger Volumes, to appear when the user arrives close to them. This way, the walk around the Temple of Valadier became an interactive tour narrating the history of the place and the surrounding territory.

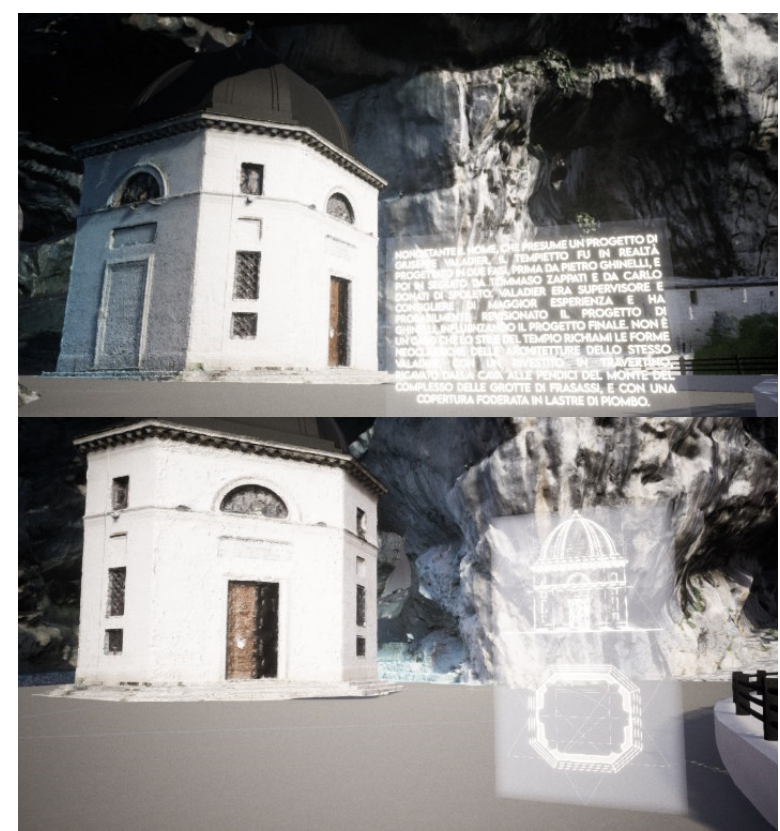

Figure 9: Few steps after the beginning of the exploration, users find the first holographic pop-up descriptions and images, becoming familiar with the scene.

The observer's vision was further treated, through a PostProcessVolume, in order to improve the final aspect of the scene and make it more interesting. The exposure compensation between the inside and the outside was adjusted to simulate the same stabilizing process of the human eye; saturation and contrast values were also corrected. In addition, other effects were set on the user view, as it was for the depth of field blur, lens flare and a delicate superficial glow in overexposed areas, in order to make the appearance of the scene more dynamic and interesting.

The application was designed to be usable both on laptop platforms and through HMDs, to make its use more versatile even in the absence of advanced devices.

At the beginning, right before getting immersed into the scene, a short introduction cinematic was implemented to give a first aerial look at the landscape of the interactive virtual walk, moving slowly and silently through the spaces around the Temple of Valadier (Fig. 10). From here, users enter the scene immersively, starting their journey from the gate at the beginning of the path, to investigate the cave, the Temple of Valadier and the landscape of the Gola di Frasassi.

\section{RESULTS}

By bringing the Captured Reality of the area of Temple of Valadier into Unreal Engine 4.27, it was possible to create an immersive and interactive experience in Virtual Reality, trying to balance the entertainment aspect with the information transmission on cultural heritage.

Users become part of a virtual environment in real-time and, thanks to the use of an HMD, can experience from the inside the landscape enclosed in the cave, confronting the proportions and visual sensations given by an alternative visit of a real site. Furthermore, using earphones, visitors can listen to a sampled replica of the real soundscape, increasing the level of sensory involvement.

The limitations of the technological resources available involved some constraints aimed at guaranteeing a stable and fluid usability of the virtual reality created. First, there was the need to decimate the photogrammetric models, partially sacrificing the high resolution of the photorealistic scene; fortunately, textures helped maintaining the impression of a high level of detail, although it was bidimensional. In addition, the absence of an aerial photogrammetric survey, and the use of legacy data, required more time and work to achieve the goal of a functional model for Virtual Reality. Future experimentations could complete the captured reality of the area, further improving the photorealism of the simulation. The introduction of the internal survey of the Temple could also be involved, which would certainly complete the spatial knowledge of the place, introducing new possibilities of interaction between users and the scenario.

Looking at the storytelling of the virtual landscape created, a suggestion to improve the user's journey, making it even more direct and immersive, could the introduction of additional sensory elements and stimuli. There could be a voiceover or a narrating character that makes the approach to the site more dynamic and engaging, giving direct inputs on what to do, without undermining their freedom of investigation, which remains one of the fundamental keys of dissemination through this medium.

Since the landscape is an everchanging multi-sensory system, it is certainly not easy to convey the totality of the real physical experience of the area, because it would require advanced and less available devices and technologies to work. However, it might be interesting to give the visitor the opportunity to experience the cave of the Temple of Valadier in various situations, perhaps by implementing different times of the day and several atmospheric conditions, transmitting more nuances 
of the same reality. The user could freely select how to see the environment, or it could be more naturally told through the introduction of a fictional story in support of communication, merging entertainment and information even more. From the storytelling point of view, possibilities are unlimited and can generate numerous alternative ways of experiencing the virtual site of the Temple of Valadier.

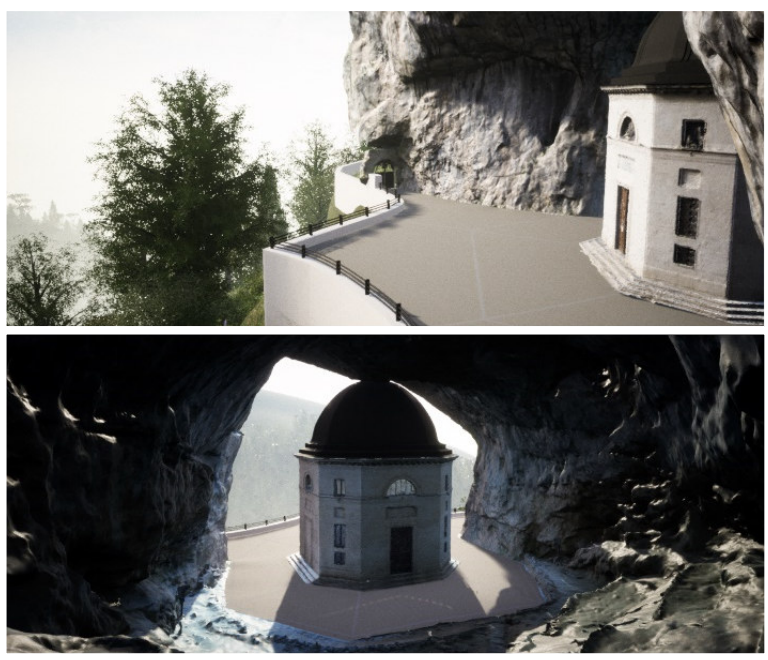

Figure 10: Frames from the opening cinematics. The landscape surrounding the Temple of Valadier highlights the strong natural qualities of the site.

\section{CONCLUSIONS}

Digital reconstruction can run into a series of critical issues, starting with technical difficulties such as the availability of adequate resources and tools. Furthermore, the very definition of cultural landscape emphasizes a series of founding characteristics that transcend the material and detectable field; this highlights the partiality of the virtual experience. An example of this is the difficulty in reproducing sensory phenomena aside from the optical and acoustic ones, which nevertheless concur to shape the perception of the place. In discussing virtual reality applied to the dissemination of cultural heritage, it is therefore essential to evaluate the great opportunities offered by new technologies in relation to the possible results that can be obtained, as well as the limits that this approach brings with it.

The particular site within the unique scenery of the Gola della Rossa is an example of how difficult and complex it is to analyse and survey a cultural heritage made up of both tangible and intangible entities at the same time. The approach to contexts of this type, including the landscape heritage, must include particular attention in the acquisition of data and in their communication, in order to recreate a virtual space that is certainly discretized but exhaustive in its complexity during an immersive experience of the user. Research in this sector has increased in recent years, but there is still no planned workflow that dictates the foundations for communicating VR environments. The choices are made autonomously and independently, and it is always in balance between a serious game with false scenarios and an excessively playful-fantasy reproduction of the virtual space. Being able to find the right representation and expression of the environment is a goal that we hope to reach, experimenting and (sometimes) making mistakes, even through more isolated and minor cases such as the Grotta della Beata Vergine, the Temple of Valadier, and the hermitage of Santa Maria Infra Saxa.

\section{REFERENCES}

Battini, C., 2017. Realtà virtuale, aumentata e immersiva per la rappresentazione del costruito. Firenze: Altralinea Edizioni.

Balletti, C., Beltrame, E., Costa, E., Guerra, F., Vernier, P., 2016. 3D reconstruction of marble shipwreck cargoes based on underwater multi-image photogrammetry. Paper in: "Digital Applications in Archology and Cultural Heritage", vol. 3.

Bolter, J. D., Grusin, R., 2000. Remediation: Understanding New Media. Cambridge, MA: MIT Press.

Butcher, J., 2017. Storytelling for Virtual Reality: Methods and Principles for Crafting Immersive Narratives. Londra: Routledge Editor.

Calisi, D., 2015. Luce e ombra nella rappresentazione. Rilettura storica e sperimentazioni eidomatiche. Roma: Aracne Editrice.

Calisi, D. Molinari, M., 2019. Patrimoni Nascosti. Documentazione e catalogazione del Tempietto di Valadier nella Gola della Rossa, Genga. In La representación grafica de naturaleza técnica (pp.27-35). Buenos Aires: Tandil.

De Luca, L., 2011. La fotomodellazione architettonica. Rilievo, modellazione, rappresentazione di edifici a partire da fotografie. Flaccovio Dario Editore.

Demestrscu, E., d'Annibale, E., Federani, D., Fanini, B., 2020. Digital replica of cultural landscapes: An experimental realitybased workflow to create realistic, interactive open world experiences. Paper in: Journal of Cultural Heritage. vol. 41.

Greengard, S., 2019. Virtual Reality. Cambridge (USA): MIT Press Edition.

Latour, B., Lowe, A., 2011. The migration of the aura - or how to explore the original through its facsimiles. In: Bartscherer, T., Coover, R. (Eds.), Switching Codes: Thinking through Digital Technology in the Humanities and the Arts. University of Chicago Press, Chicago, MA, pp. 275-297.

Regione Marche, 2000. Scheda n. 39 "Gola di Frasassi". Aree floristiche protette dalla L.R. 52 del 30/12/1974.

Monti, C., Selvini, A., 2015. Topografia, fotogrammetria e rappresentazione all'inizio del ventunesimo secolo. Milano: Maggioli Editore.

Pietroni, E., Adami, A., 2014. Interacting with Virtual Reconstructions in Museums: The Estruscanning Project. Paper in: ACM Journal on Computing and Cultural Heritage, vol. 7, n. 2, pp. 1-29.

Riccò, D, 2008. Sentire il design. Sinestesie nel progetto di comunicazione. Roma: Carrocci Editore.

Riva, G., Gaggioli, A., 2019. Realtà virtuali. Gli aspetti psicologici delle tecnologie simulative e il loro impatto sull'esperienza umana. Firenze: Giunti Psychometrics. 